gradual preparation of elaborate maps of the German and Austrian Alps. At present it is engaged on a map of the Salzkammergut, on a scale of I : 100,000.

\section{A LUNAR LANDSCAPE}

MESSRS. GAMMON AND VAUGHAN, No. 28, Old Bond Street, have at present on view a picture in which the artist, Olafs Winkler, of Weimar, has endeavoured to represent a lunar landscape. Prof. C. Bruhns, of Leipzig, has assisted him in the parts of the treatment which are directly scientific.

The painter has not trusted all to his imagination. He has, to the best of his knowledge and ability, sought to stick rigidly to truth, and to paint a lunar landscape such as it would appear, so far as human observation has hitherto ascertained, to a human eye, were it at all possible for a man to be transplanted to the moon and observe through his earthly eyes, only for a moment, nature as she manifests herself on the surface of our satellite. From the merely artistic point of riew the artist fears his task may be a thankless one, for since the moon has no atmosphere, there is neither aërial perspective nor diffusion of light, but it is precisely this point which should make our artist all the more interested in this unique production. The shadow of a body in the foreground will appear quite as black as the sky itself which closes the landscape like a flat steep wall, broken only by the quiet light of the stars. All lights appear equally strong at a distance and close at hand, and this also holds with the local colouring. In a word, there is wanting in the lunar landscape that which lends to our earth perspective, richness or tone, modulation, softness, and temper. It is our atmosphere we have to thank for most of the multitudinous coloured phenomena of the terrestrial landscape-phenomena which in our satellite are impossible. The sunlight falls upon the hills with blinding brightness, and cuts sharply across the deep black shadows. Its intensity rivals the electric light, and light effects of such a kind are far beyond the reach of our palettes. We must resort to some expedient to be able to introduce a medium between the extreme contrast of light and shade, a sort of half-tone, which, at the same time, must be the chief tone of the picture; this Herr Winkler has sought in the light of the earth, the true "earthshine."

The artist has chosen the time of sunset, and the region he has selected lies in the northern part of the moon. The spectator is supposed to be on the front slope of a mountain, the continuation of which in the background comes out as a closed ridge. At his feet one of the numerous maria spreads out, filled up with rills, circular hills, and large and small craters, stretching away to the distant mountain referred to. Before, us in the black sky, hangs the moon's moon, our earth. She sheds her pale, ash-coloured light over the rent, desolate, dead stone-fields. Only the highest points of the mountain-tops still glow in the light of the setting sun, no longer red, as here, but dazzlingly white, in consequence of the absence of atmospheric absorption. The earth is at the period of her course between Sagittarius and the Scorpion, Antares being nearly in the middle of the picture. Against his persuasion he has been compelled to make the milky way very weak, and the stars somewhat large in proportion to the earth.

Herr Winkler, in a paper read at the last meeting of the German Association, stated that his first impulse to undertake the picture was derived from Nasmyth and Carpenter's work on the moon.

Our only criticism of the picture refers to the colour of the earth and of the true earthshine. We doubt whether the earth is quite red enough, especially at the edges, and we doubt again whether, with the earth as ruddy as it is, the colour of the lunar landscape itself should not be rathermore in harmony with it, as it is the true light
source.

The picture is an admirable performance, and the science of it is so true that, as we hinted before, those of our artists who care to have a natural basis for their depiction of natural phenomena will learn much from this attempt to deal with a new order of phenomena.

\section{EDISON'S TALKING-MACHINE'}

$\mathrm{M}^{\mathrm{R}}$ R. THOMAS A. EDISON has recently invented an instrument which is undoubtedly the acoustic marvel of the century. It is called the "Speaking Phonograph," or, adopting the Indian idiom, one may aptly call it "The Sound-Writer who Talks." Much curiosity has been expressed as to the workings of this instrument, so I purpose giving an account of it.

All talking-machines may be reduced to two types. That of Prof. Faber, of Vienna, is the most perfect example of one type; that of Mr. Edison is the only example of the other.

Faber worked at the source of articulate sounds, and built up an artificial organ of speech, whose parts, as nearly as possible, perform the same functions as corresponding organs in our vocal apparatus. A vibrating ivory reed, of variable pitch, forms its vocal chords. There is an oval cavity, whose size and shape can be rapidly changed by depressing the keys on a key-board. A rubber tongue and lips make the consonants; a little windmill, turning in its throat, rolls the letter $r$, and a tube is attached to its nose when it speaks French. This is the anatomy of this really wonderful piece of mechanism.

Faber attacked the problem on its physiological side. Quite differently works Mr. Edison: he attacks the problem, not at the source of origin of the vibrations which make articulate speech, but, considering these vibrations as already made, it matters not how, he makes these vibrations impress themselves on a sheet of metallic foil, and then reproduces from these impressions the sonorous vibrations which made them.

Faber solved the problem by reproducing the mechanical causes of the vibrations making voice and speech; Edison solved it by obtaining the mechanical effects of these vibrations. Faber reproduced the movements of our vocal organs; Edison reproduced the motions which the drum-skin of the ear has when this organ is acted on by the vibrations caused by the movements of the vocal organs.

Figs. I and 2 will render intelligible the construction of Mr. Edison's machine. A cylinder, F, turns on an axle which passes through the two standards, $A$ and $B$. On one end of this axle is the crank, D; on the other the flywheel, $\mathrm{E}$. The portion of this axle to the right of the cylinder has a screw-thread cut on it, which, working in a nut, $A$, causes the cylinder to move laterally when the crank is turned. On the surface of the cylinder is scored the same thread as on its axle. At $F$ (shown in one-half scale in Fig. 2) is a plate of iron, A, about $\frac{7}{100}$ of an inch thick. This plate can be moved toward and from the cylinder by pushing in or pulling out the lever $\mathrm{H} \mathrm{G}$, which turns in an horizontal plane around the pin $\mathrm{J}$.

The under side of this thin iron plate, A (Fig. 2), presses against short pieces of rubber tubing, $\mathrm{x}$ and $\mathrm{X}$, which lie between the plate and a spring attached to $\mathrm{E}$. The end of this spring carries a rounded steel point, $\mathrm{P}$, which enters slightly between the threads scored on the cylinder c. The distance of this point, $P$, from the cylinder is regulated by a set-screw, s, against which abuts the lever, H G. Over the iron plate, A, is a disc of vulcanite, B B,

I The figures in this article are taken from "Sound, a Series of Simple, Entertaining, and Inexpensive Experiments in the Phenomena of Sound, for the Use of Students of every Age." By Alfred M. Mayer. Vol. ii. of "Experimental Science Series for Beginners," (Now in press and soon to be published by $D$. Appleton and Co.) 